\title{
Necrotizing fasceitis post cesarean section
}

\begin{abstract}
Necrotizing Fasceitis (NF) is a subcutaneous soft tissue infection very few frequent but very serious for the patient's health. We present a case of a young pregnant woman who underwent to a cesarean section by medical reasons related with lack of evolution of labor and unexpectedly developed NF at $12 \mathrm{hs}$ of post operative evolution and a literature revision about this subject. In all cases, the only probability to solve the situation (and not always is achieved) is by a very fast surgical and medical treatment by a multidisciplinary trained team.
\end{abstract}

Keywords: Necrotizing fasceitis, maternal infection, septic shock, surgical remotions

\author{
Volume 6 Issue I - 2018 \\ R Illia,' R Durlach, ${ }^{2}$ Bugari G, ${ }^{3}$ P Olejnik ${ }^{4}$ \\ 'Professor of Obstetrics, Buenos Aires University, Argentina \\ ${ }^{2}$ Professor of Infectology, Catholic University, Argentina \\ ${ }^{3}$ Staff member of General Surgery Service, Hospital Aleman, \\ Argentina \\ ${ }^{4}$ Staff member of Obstetrics Service, Hospital Aleman, Argentina
}

Correspondence: R Illia, Professor of Obstetrics at Buenos Aires University, FACOG, FRSM, Chief of Obstetrics Service at Hospital Aleman, Buenos Aires, Argentina,

Email rhillia@gmail.com

Received: February 08, 2018 | Published: March 02, 2018

\section{Introduction}

Necrotizing Fasceitis (NF) is a subcutaneous soft tissue infection very few frequent but very serious for the patient's health. It consist in a subcutaneous bacterial infection that occur after a surgical incision or an accidental skin wound. It was recently shown that most of the disseminate skin infections included NF, are originated by bacterial mixed infections that act synergically. This evidence was seen in Clinical and experimental studies. ${ }^{1}$ This multi bacterial infection, generally happen in patients with predisponent factors to bacterial inoculation and to dissemination of the disease. ${ }^{2}$ The diagnosis of $\mathrm{NF}$ require the intra surgical identification of necrotic fascia in a patient with fever. ${ }^{9}$ Goepfert et al. ${ }^{3}$ have communicated a rate of 1.8 cases every 1000 cesarean sections (CS). Mabie et al. ${ }^{4}$ have studied 18 patients with septic shock during pregnancy. In $30 \%$ of cases, the origin was pielonephritis and one patient suffered NF.

\section{Clinical case}

A 23 years old patient was admitted in a latent prelabor with a 41 pregnancy week's of gestational age. As obstetrical background, she had two previous pregnancies that ended in spontaneous abortions. She did the prenatal controls without any kind of complications. At admission, the cervix was without significant modifications and $1 \mathrm{~cm}$ of dilation. The cervical maturation with vaginal dinoprostone was started. The active phase was achieved, but with a cervical dilation of $4 \mathrm{~cm}$, the fetal heart rate presented a non reassuring pattern and a CS was performed. It was delivered after intrauterine reanimation a vigorous fetus with a weight of $3080 \mathrm{~g}$, Apgar 9/10. At 24hs after CS, the patient started with abdominal distension and scarse gut movements. The oral hydration was gave up and started with paraenteral hydration. Twelve hours after, the patient present an important abdominal distension. The abdominal X-ray showed us a distension of right colon and a nasogastric catheter was introduced. The same day, we evaluated the patient with the surgeons. The patient does not present gut activity, there was peritoneal irritability with abdominal defense at palpation, specially at the right place of abdomen. At abdominal right side, we observed equimosis and inflammatory sign. A new abdominal
$\mathrm{X}$-ray showed a large colon distension and a cecal diameter of more than $10 \mathrm{~cm}$. The blood pressure was $100 / 50$ and the pulse weak. It was decided to perform an exploratory laparotomy under suspicion of abdominal and abdominal wall infection with systemic maternal compromise. During the laparotomy it was observed purulent and necrotic material with muscular and aponeurotic layers.

Once open the abdominal cavity, it was obtained seropurulent fluid. The necrotic area compromiso the aponeurotic layer and both anterior muscles of abdomen. It was done a wide resection of the necrtotic tissues, cleaning of abdominal cavity and drainages in below liver space, in right and left parietocolic places and in Douglas space. Besides, there were put two tubes in subaponeurotic space. With a diagnosis of severe infections of soft tissues and peritonitis, the patient was taken to Intensive Care Unit (ICU) under medication with endovenous penicillin until the outcomes of the cultures because of suspicion of Clostridium sp infection. Three hours later, the culture informed positive coccus in all samples obtained and it was started a three antibiotic treatment with penicillin, gentamycin and metronidazol. At $24 \mathrm{hs}$ of surgery, the injury progressed toward cellulite in the right abdominal place with blisters. It was done a surgical toilette and the laboratory informed the outcome of the culture: beta hemolityc streptococcus. The patient remain in ICU with oliguria, the chest $\mathrm{X}$ ray informed bilateral heterogeneous opacity, she developed bronchoespasm and low blood pressure and underwent to mechanical respiratory assistance because respiratory acute failure with hipoxia and metabolic acidosis. The antibiotic treatment is changed toward penicillin, gentamycin and rifampycin. She continue with respiratory distress, septic liver and acute kidney insufficiency with a creatinine level of $1.22 \mathrm{mg} \%$. The patient continued with daily toilettes of the necrotic areas. The final outcome of the culture was: betha hemolitic streptococcus Group A. Four days later, the patient presented multiorganic failure with encephalopathy. We do not know why, but from this moment, may be because the change in the antibiotic treatment, the patient started to doing better. The endotracheal tuve was removed, the necrotic areas progressed lesser tan bedore, but the patient continued with the schedule of daily remotions of necrotic 
tissues. Fifhteen days later, the gentamycin was gave up and the patient could leave ICU. Ten days later, she was discharged from Infectology Service and about the month of CS she start with the plastic surgeries. Finally, 70 days later of CS, she was discharged. The sequence of cultures was: immediatly post CS: beta hemolitic Streptococcus Group A, ten days later: Pseudomona aeruginosa \& Staphylococcus aureus MR, by the month: enterobacteriae cloacae and the week after to be admitted in ICU: Klebsiella Pneumoniae from the subclavial catheter.

\section{Comments}

NF include two bacterial entities; type I and II. In the type I, at least one bacteria cultured is anaerobic (most frequently Bacteroides $s p$. or Peptoestreptococcus $s p$ ) combined with one or more anaerobic facultative specimen such as Streptococci (different from type A) and Enterobacteriaceae (like E. Coli, Enterobacter sp., Klebsiella sp., Proteus $s p$ ). The cases with infections produced by one bacteria or only involve anaerobics are weird. In the type II, which may have as synonymous streptococcic gangrene, the responsible bacteria is Streptococcus group A B-haemolityc or S. Pyogenes.

This entity could appear as a one bacterial infection or mixed associated with Streptococcus aureus or others. In the invasive disease by Streptococcus pyogenes, the picture of fever, shock and multiorganic failure is indistinguishable from the disease produced by negative Gram bacteria or mixed infections. Like in the infections by negative Gram the LPS of the bacterial wall is the antigen that start the inflamatory cascade, in the disease by S. Pyogenes is a toxin named eritrogenic toxin with three serotypes: Spe A, B and C. The eritrogenic toxin and the TSST-1, have the properties of a super antigen, both of them increase the growth of $T$ cells and produced high levels of cytoquines and tumoral necrosis factor. The toxin Spe from Streptococcus is more virulent than the toxin from S. Aureus. The virulence could be associated to the strategic capability to avoid phagocytosis.

The presentation of fever during puerperium is a common complication. Although the primary cause of fever is the genital zone, it is required a careful evaluation of the puerperal patient with fever before start treatment with antibiotics because the right handle of this situations would decrease the morbimortality associated to puerperal infections. ${ }^{5}$ Schorge et $a .^{6}$ communicated about their experience with 17 patients with NF. They conclude that the early diagnosis and agressive surgical remotion of necrotic tissue, may improve prognosis. This opinion, coincide with the Mabie et $\mathrm{al}^{4}$ research in which they underscore that in patients with septic shock, the progression to die could be dramatically fast. According the increase in the vascular permeability, it could be appropiate administrate vasopressors earlier during the resuscitation maneuvers. A low heart work is a bad prognosis factor. McHenry et al. ${ }^{2}$ communicated three cases of microbial NF, two cases by Streptococcus pyogenes and one case by Stafilococcus aureus. All the patients came with acute infection with fast evolution and one of them died for sepsis. The authors cosider that in this kind of cases, the bacterial virulence and inmunological response of the host could have been influenced for changes in inmunological response produced by pregnancy. In the Goepfert et al revision, ${ }^{3}$ all the patients with NF underwent to several surgical remotions of necrotic tissues and received wide spectrum antibiotics.
Piper et al. ${ }^{7}$ communicated one case which developed four days after a postpartum tubal ligation. The patient evolution was satisfactory based in antibiotics and frequent necrotic tissue remotions. Mathews et al. ${ }^{8}$ informe done case of NF after cesarean section produced by Apophysomyces elegans. Again, the antibiotic treatment (in this case with anphotericin B) and repeated tissue remotions, contributed to the patient good evolution. It is very clear that NF diagnosis require a high level suspicion because some subtle initial signs could be underestimated. In front of a puerperal patient who present a torpid evolution with fever, is the most importance to think about the possibility of a NF and fastly search for subtle signs of their presence. If the suspicion have fundament, specially because the fast worsening of the patient state and the observation of associated skin lesions, only it will be posible to have a chance if a fast and agressive surgical treatment is started with repeated remotions of necrotic tissues associated to right antibiotic treatment.

Probably, as $\mathrm{McHenry}^{2}$ affirm, the patient would be a Carrier of conditionant factors like immunodepresion, attributable to pregnancy, or for the presence of other intercurrences like associated diseases, under nourishment, drug adictions, etc. In all cases, the only probability to solve the situation (and no always is achieved) is by a very fast surgical and medical treatment by a multidisciplinary trained team.

\section{Acknowledgements}

None.

\section{Conflict of interest}

Authors declare there is no Conflict of interest towards this manuscript.

\section{References}

1. Martinez Velasco I, Plascencia Valadez V, Salinas Trevino J, et al. Post cesarean NF. Bacterial sinergismo or mixed infection of soft tissues?

2. McHenry C, Azar T, Ramahi AJ, et al. Monomicrobial necrotizing fasciitis complicating pregnancy and puerperium. Obstet Gynecol. 1996;87(5 Pt 2):823-826

3. Goepfert A, Guinn D, Andrews WW, et al. Necrotizing fasciitis after cesarean delivery. Obstet Gynecol. 1997;89(3):409-412.

4. Mabie W, Barton J, Sibai B. Septic shock in pregnancy. Obstet Gynecol. 1997;90(4 Pt 1):553-561.

5. Calhoun B, Brost B. Emergency management of sudden puerperal fever. Obstet Gynecol Clin North Am. 1995;22(2):357-367.

6. Schorge J, Granter S, Lerner LH, et al. Postpartum and vulvar necrotizing fasciitis. Early clinical diagnosis and histopathologic correlation. J Reprod Med. 1998;43(7):586-590.

7. Piper J, West P. Necrotizing fasciitis following postpartum tubal ligation. A case report and review of the literature. Arch Gynecol Obstet. $1995 ; 256(1): 35-38$.

8. Mathews M, Raman A, Nair A. Nosocomial zygomycotic post-surgical necrotizing fasciitis in a healthy adult caused by Apophysomyces elegans in south India. J Med Vet Mycol. 1997;35(1):61-63.

9. Dennis L Stevens, Amy E Bryant. Necrotizing Soft-Tissue Infections. $N$ Engl J med. 2017;377(23):2253-2265. 